\title{
PROJETO DE OBSERVADORES DE FLUXO PARA O CONTROLE POR ORIENTAÇÃO DIRETA DO CAMPO EM MÁQUINAS DE INDUÇÃO PENTAFÁSICAS
}

\author{
Micaela Benavides* \\ micaeletro@gmail.com
}

Daniel Ferreira Coutinho $\ddagger$

coutinho@das.ufsc.br

\author{
Luís Fernando Alves Pereira ${ }^{\dagger}$ \\ lapereiradece.ufrgs.br
}

\author{
Luís Alberto Pereira ${ }^{\S}$ \\ lpereiraepucrs.br
}

\footnotetext{
*Grupo de Automação e Controle de Sistemas, Programa de Pós-Graduação em Engenharia Elétrica, Pontifícia Universidade Católica do Rio Grande do Sul, Av Ipiranga, 6681, 90619-900, Porto Alegre, RS, Brasil.

${ }^{\dagger}$ Grupo de Controle, Automação e Robótica, Programa de Pós-Graduação em Engenharia Elétrica, Universidade Federal do Rio Grande do Sul, Av. Osvaldo Aranha 103, 90035-190, Porto Alegre, RS, Brasil.

${ }^{\ddagger}$ Grupo de Automação e Controle de Sistemas, Programa de Pós-Graduação em Engenharia Elétrica, Pontifícia Universidade Católica do Rio Grande do Sul, Av Ipiranga, 6681, 90619-900, Porto Alegre, RS, Brasil.

${ }^{\S}$ Grupo de Automação e Controle de Sistemas, Programa de Pós-Graduação em Engenharia Elétrica,

Universidade Federal do Rio Grande do Sul, Av. Osvaldo Aranha 103, 90035-190, Porto Alegre, RS, Brasil.
}

\section{ABSTRACT}

Design of Flux Observers for Direct Field Control in Five Phase Induction Machines

This paper proposes a convex optimization approach for the design of robust observers applied to the control of five phase induction machines subject to rotor resistance variation and load disturbance. Based on the first and third harmonic dynamic models of the multi-phase machine, two robust observers are proposed to estimate the rotor flux components. The two observers are applied to the direct field oriented control of a five phase induction machine, where simulation results have demonstrated a high dynamic performance and robustness to parametric variations of the proposed setup.

KEYWORDS: Five Phase Induction Machines, Robust Observer, Direct Field Oriented Control.

Artigo submetido em 24/01/2010 (ld.: 01099)

Revisado em 29/03/2010, 20/08/2010

Aceito sob recomendação do Editor Associado Prof. José Antenor Pomilio

\section{RESUMO}

Apresenta-se neste artigo a aplicação de técnicas de otimização convexa para o projeto de observadores robustos aplicados a motores de indução pentafásicos, sujeitos a variações na resistência do rotor e a perturbações de carga. É desenvolvido um estimador robusto para os fluxos do primeiro e terceiro harmônico do rotor, cuja validação é realizada junto ao controle por orientação de campo no modo direto admitindo aplicações que exijam elevado desempenho dinâmico e alta robustez frente a variações paramétricas e distúrbios externos.

PALAVRAS-CHAVE: Máquinas de Indução Pentafásicas, Observadores Robustos, Controle por Orientação Direta do Campo

\section{INTRODUÇÃO}

Com a evolução tecnológica na área de eletrônica de potência e o consequente desenvolvimento dos conversores de 
frequência, tornou-se viável o emprego dos motores de indução em aplicações onde são necessárias velocidades variáveis. A forma de controle mais simples destes motores, conhecida como controle escalar, baseia-se na variação da relação entre a frequência e a tensão aplicadas ao motor (relação V/F). Embora útil para aplicações de velocidade variável, o controle $\mathrm{V} / \mathrm{F}$ apresenta restrições para operação em baixas velocidades e também um pobre desempenho dinâmico. Com o objetivo de tornar o desempenho dinâmico do motor de indução comparável aos dos motores de corrente contínua, no trabalho desenvolvido por Blaschke (Blaschke, 1972) foi apresentado a proposta do controle por orientação do campo. No controle por orientação do campo, considera-se o modelo do motor representado em coordenadas direta e de quadratura operando no referencial síncrono, estabelecendo-se condições para que o fluxo do rotor seja manipulado pela corrente de eixo direto do estator, sendo o torque do motor manipulado pela corrente de quadratura do estator, possibilitando o desacoplamento entre o fluxo do rotor e o torque eletromagnético do motor de maneira similar às máquinas de corrente contínua.

Atualmente, o controle por orientação do campo em máquinas de indução passou a ser largamente utilizado, conferindo a estas máquinas características de alto desempenho dinâmico e sua utilização nas mais diversas aplicações. Uma vez que o controle por orientação do campo necessita de inversores de frequência para o acionamento de máquinas de indução, a exigência de um número específico de fases passou a não ser mais essencial, e um número maior que três pode ser escolhido (Toliyat, 1998). Sob esse aspecto, iniciaram-se estudos sobre os possíveis benefícios da utilização de motores de indução com número de fases superior a três (Moreira e Lipo, 1992), (Lyra e Lipo, 2002), (Jacobina et al., 2004). Uma análise das diferentes opções de número de fases (três, seis, cinco, sete e nove fases) no projeto de máquinas de indução para uso em aplicações de velocidade variável é visto em (Toliyat et al., 1991). Neste estudo é feita uma análise das alternativas de enrolamentos que permitam distribuições de campo trapezoidais ou retangulares com o uso de inversores. Sob o ponto de vista de produção de torque, chegou-se a conclusão que o motor de indução pentafásico associado a um sistema de acionamento capaz de produzir uma distribuição de campo próxima do retangular apresentava os melhores resultados (Scharlau et al., 2008). Da mesma forma que os motores de indução trifásicos, motores de indução pentafásicos apresentam comportamento dinâmico não-linear, o fluxo magnético que deve ser controlado não é diretamente mensurável e alguns parâmetros são variantes no tempo, em especial a resistência do rotor pode sofrer uma alteração de até $100 \%$ do seu valor nominal com a variação da temperatura e pela saturação do meio magnético (Prempain e Postlethwaite, 2002). Além disso, geralmente o torque solicitado pela carga pode sofrer uma variação relativamente grande (a vazio e a plena carga) o que torna o controle do motor de indução uma tarefa difícil em aplicações onde é necessário alto desempenho dinâmico.

O controle por orientação do campo pode ser realizado de duas formas, conhecidas como controle por orientação indireta do campo e como controle por orientação direta do campo. Na forma indireta, o modelo do motor é utilizado para determinar o escorregamento entre os campos magnéticos do estator e do rotor. $\mathrm{O}$ cálculo deste escorregamento depende da constante de tempo do rotor que é afetada pela temperatura e pela saturação do meio magnético. Na forma direta, é necessário medir ou estimar o fluxo do rotor utilizado para efetuar a conversão entre os sistemas de coordenadas estacionárias e síncronas, sendo possível desacoplar o controle de fluxo do controle de torque do motor. A determinação dos fluxos pode ser feita por observadores de estado ou algoritmos adaptativos baseados em filtros de Kalman (Petersen e Savkin, 1999), (Salvatore et al., 1993), (Pereira et al., 1998) ou em observadores de Luenberger (Luenberger, 1966). Trabalhos recentes têm aplicado técnicas de otimização convexa empregando desigualdades matriciais lineares (ou LMIs) no projeto de observadores robustos para motores de indução (Coutinho, Curcio, Pereira e Haffner, 2004), (Coutinho et al., 2007).

Desde o desenvolvimento dos métodos de pontos interiores para a solução de problemas de programação semi-definida, técnicas baseadas em LMIs tem sido largamente utilizadas na solução de diferentes problemas na teoria de controle. A idéia básica é a de reformular o problema de controle em termos de desigualdades matriciais simétricas e afins nas variáveis de decisão. Novos avanços na aplicação das técnicas LMI podem ser encontradas em (Ghaoui e Niculescu, 2000), como por exemplo análise de estabilidade e síntese de controladores para sistemas lineares a parâmetros variantes, otimização de restrições integrais quadráticas (IQCs), sistemas impulsivos, ganho variável ("gain-scheduling") e controle multi-objetivo.

A aplicação direta das técnicas LMIs em sistemas nãolineares resultam em desigualdades matriciais não-lineares (ou NLMIs) perdendo a propriedade da convexidade. Tal fato acarreta na obrigatoriedade do cálculo ponto a ponto no espaço de estados (ou um subconjunto deste) das condições de estabilidade gerando um enorme esforço computacional. Apesar desse fato, vários trabalhos na literatura de controle não-linear propõem métodos aproximados para a solução de NLMIs utilizando, por exemplo, a técnica das diferenças finitas (Huang e Lu, 1996). Recentemente, em (Coutinho et al., 2002), (Coutinho e Trofino, 2002) e (Coutinho, Fu e Trofino, 2004), foram apresentados resultados relacionados a formulação convexa de LMIs não-lineares, permitindo a aplicação de diversas técnicas LMIs na análise de estabilidade e 
síntese de controle para sistemas não-lineares incertos, dentro os quais podem se encaixar os modelos dos motores de indução.

Apresenta-se neste artigo a extensão do observador robusto não-linear para a estimativa dos fluxos do rotor em máquinas de indução trifásicas, apresentado em (Coutinho et al., 2007), para máquinas de indução pentafásicas empregando o modelo desacoplado descrito em termos das variáveis simétricas de valor instantâneo para o primeiro e terceiro harmônico, apresentado em (Pereira et al., 2010), admitindo variação na resistência do rotor e distúrbios na carga mecânica acoplada ao eixo rotor da máquina. $\mathrm{O}$ vetor de ganhos do observador não linear é projetado de forma a minimizar o limite superior do ganho $\mathcal{L}_{2}$ da relação entre o distúrbio de carga e o erro de estimação dos fluxos do rotor para toda a faixa admissível de variação da resistência do rotor. Os fluxos observados são utilizados para o controle por orientação direta do campo empregando as componentes fundamental e de terceiro harmônico do fluxo do rotor. Os resultados obtidos com o observador são comparados com os resultados obtidos utilizando os fluxos calculados com modelo empregado na simulação da máquina de indução pentafásica.

\section{LISTA DE SÍMBOLOS}

$v_{k}^{s}$ - tensão aplicada na fase $k$ do estator;

$\widetilde{v}_{l}^{s}$ - tensão de sequência $l$ do estator;

$i_{k}^{s}$ - corrente da fase $k$ do estator;

$i_{k}^{r}$ - corrente da fase $k$ do rotor;

$\widetilde{i}_{l}^{s}$ - corrente de sequência $l$ do estator;

$\widetilde{i}_{l}^{r}$ - corrente de sequência $l$ do rotor;

$\widetilde{i}_{l}^{r s}$ - corrente de sequência $l$ do rotor referenciado ao estator;

$\omega_{e}$ - velocidade do rotor em graus elétricos/s;

$\omega_{m}$ - velocidade do rotor em graus mecânicos/s;

$T_{e}$ - torque eletromagnético da máquina;

$T_{l}$ - torque de carga;

$J_{m}$ - coeficiente de atrito viscoso;

$B_{m}$ - coeficiente de amortecimento do conjunto motor e carga;

$\Psi_{k}^{s}$ - fluxo concatenado com a fase $k$ do estator;

$\Psi_{k}^{r}$ - fluxo concatenado com a fase $k$ do rotor;

$R_{k}^{s}$ - resistência da fase $k$ do estator;
$R_{k}^{r}$ - resistência da fase $k$ do rotor;

$R_{b}^{r}$ - resistência de uma barra do rotor;

$\widehat{L}_{n}^{r s}$ - valor máximo da indutância mútua entre uma fase do estator e uma fase do rotor para o harmônico de ordem $n$;

$\phi$ - ângulo de deslocamento entre o eixo da fase 1 do estator e da fase 1 do rotor;

$p$ - número de pares de polos;

$\widetilde{R}^{s}$ - resistência de sequência do estator;

$\widetilde{L}_{j}^{s}$ - indutância de sequência $j$ do estator;

$\widetilde{R}_{j}^{r}$ - resistência de sequência $j$ do rotor;

$\widetilde{L}_{j}^{r}$ - indutância de sequência $j$ do rotor;

$m_{s}$ número de fases do estator;

$m_{r}$ número de fases do rotor.

Notação: $\mathbb{R}^{n}$ denota o conjunto de vetores reais $n$ dimensionais, $\mathbb{R}^{n \times m}$ denota o conjunto de matrizes reais de ordem $n \times m, I_{n}$ é uma matriz identidade de dimensão $n \times n$, $0_{n \times m}$ é uma matriz de zeros de ordem $n \times m, 0_{n}$ é uma matriz de zeros de ordem $n \times n$, e $\operatorname{diag}\{\ldots\}$ representa uma matriz bloco diagonal. Para matrizes $S, S^{T}$ denota sua transposta e $S>0$ indica que $S$ é positiva definida. Para blocos de matrizes, o símbolo $\star$ significa a existência de blocos simétricos transpostos fora dos blocos da diagonal principal. Para os politopos $X_{1} \subset \mathbb{R}^{n}$ e $X_{2} \subset \mathbb{R}^{m}$, a notação $X_{1} \times X_{2}$ significa que $\left(X_{1} \times X_{2}\right) \subset \mathbb{R}^{(n+m)}$ representa um meta politopo obtido pelo produto cartesiano, e $\mathcal{V}\left(X_{i}\right)$ é o conjunto de todos os vértices de $X_{i}$. Matrizes e vetores cujas dimensões são omitidas no texto são aquelas em que tais informações podem ser inferidas diretamente pelo contexto. " " corresponde a componentes simétricas de valor instantâneo e "* simboliza complexo conjugado.

\section{MODELO DO MOTOR DE INDUÇÃO PENTAFÁSICO}

Será apresentado nesta seção o modelo matemático do motor de indução pentafásico utilizado para o projeto dos controladores de alto desempenho dinâmico, robustos a variações de parâmetros e distúrbios de torque.

No modelo do motor de indução pentafásico, detalhadamente apresentado em (Pereira et al., 2006), será considerado o efeito do harmônico fundamental e do terceiro harmônico no campo do entreferro. A importância da inclusão do efeito dos harmônicos de espaço se deve a possibilidade da geração 
de torque adicional na máquina. Para a parte elétrica da máquina são consideradas as equações das tensões para o estator e para o rotor, descritas a seguir:

$$
\begin{gathered}
{[v]^{s}=[R]^{s} \cdot[i]^{s}+[L]^{s s} \cdot \frac{d[i]^{s}}{d t}+\frac{d\left\{[L]^{s r} \cdot[i]^{r}\right\}}{d t}} \\
{[0]=[R]^{r} \cdot[i]^{r}+[L]^{r r} \cdot \frac{d[i]^{r}}{d t}+\frac{d\left\{[L]^{r s} \cdot[i]^{s}\right\}}{d t}}
\end{gathered}
$$

Para a parte mecânica, a equação que descreve o torque eletromagnético $\left(T_{e}\right)$ da máquina pode ser expressa na forma matricial como:

$$
T_{e}=\frac{p}{2} \cdot\left[[i]^{s T} \mid[i]^{r T}\right]^{*} \cdot \frac{d}{d \phi}\left[\begin{array}{l|l}
{[L]^{s s}} & {[L]^{s r}} \\
\hline[L]^{r s} & {[L]^{r r}}
\end{array}\right] \cdot\left[\frac{[i]^{s}}{[i]^{r}}\right]
$$

onde $[v]^{s}=\left[v_{1}^{s}, v_{2}^{s}, v_{3}^{s}, v_{4}^{s}, v_{5}^{s}\right]^{T}$ é o vetor de tensões do estator, $[i]^{s}=\left[i_{1}^{s}, i_{2}^{s}, i_{3}^{s}, i_{4}^{s}, i_{5}^{s}\right]^{T}$ é o vetor de correntes do estator, $[i]^{r}=\left[i_{1}^{r}, i_{2}^{r}, i_{3}^{r}, \cdots i_{15}^{r}\right]^{T}$ é o vetor de correntes do rotor, $[R]^{s}$ a matriz diagonal contendo as resistências próprias do estator, $[R]^{r}$ a matriz de resistências do rotor, $[L]^{s s}$ e $[L]^{r r}$ as matrizes de indutâncias próprias do estator e do rotor, $[L]^{s r}$ e $[L]^{r s}$ as matrizes de indutâncias mútuas entre o estator e o rotor.

As equações que descrevem o comportamento dinâmico da máquina ((1)-(3)), além de serem fortemente acopladas, não permitem avaliar a influência de cada harmônico em separado. Este sistema de equações pode ser consideravelmente simplificado através da introdução de transformações de coordenadas descrita em (White e Woodson, 1959), obtendose um novo conjunto de variáveis de tensões e correntes denominadas de componentes simétricas de valor instantâneo. Conforme detalhadamente apresentado em (Pereira et al., 2010), esta transformação é aplicada diretamente aos valores instantâneos das tensões e das correntes, não sendo feita nenhuma restrição quanto a forma de onda das mesmas.

$$
\begin{aligned}
& {[v]^{s}=[O] \cdot[\widetilde{v}]^{s} \Leftrightarrow[\widetilde{v}]^{s}=[O]^{-1} \cdot[v]^{s}} \\
& {[i]^{s}=[O] \cdot[\widetilde{i}]^{s} \Leftrightarrow[\widetilde{i}]^{s}=[O]^{-1} \cdot[i]^{s}} \\
& {[i]^{r}=[Q] \cdot[\widetilde{i}]^{r} \Leftrightarrow[\widetilde{i}]^{r}=[Q]^{-1} \cdot[i]^{r}}
\end{aligned}
$$

onde $[\widetilde{v}]^{s}=\left[\begin{array}{lllll}\widetilde{v}_{0}^{s} & \widetilde{v}_{1}^{s} & \widetilde{v}_{2}^{s} & \widetilde{v}_{3}^{s} & \widetilde{v}_{4}^{s}\end{array}\right]^{T}$ é o vetor das componentes simétricas das tensões do estator, $\left[\begin{array}{l}\tilde{i}\end{array}\right]^{s}=\left[\begin{array}{llllll}\widetilde{i}_{0}^{s} & \widetilde{i}_{1}^{s} & \widetilde{i}_{2}^{s} & \widetilde{i}_{3}^{s} & \widetilde{i}_{4}^{s}\end{array}\right]^{T}$ é o vetor das componentes simétricas das correntes do estator, $[\widetilde{i}]^{r}=\left[\begin{array}{lllll}\widetilde{i}_{0}^{r} & \widetilde{i}_{1}^{r} & \widetilde{i}_{2}^{r} & \ldots & \widetilde{i}_{14}^{r}\end{array}\right]^{T}$ é o vetor das componentes simétricas das correntes do rotor. As componentes $\widetilde{i}_{0}^{s}$ e $\widetilde{i}_{0}^{r}$ são as componentes de sequência zero da corrente do estator e do rotor e só tem valor diferente de zero quando a soma das correntes da máquina tiver um caminho de circulação.

Para o estator a transformação será definida pela matriz complexa (7), composta pelos autovetores da matriz $[L]^{s s}$,

$$
[O]=\frac{1}{\sqrt{5}} \cdot\left[\begin{array}{ccccc}
1 & 1 & 1 & 1 & 1 \\
1 & a^{-1} & a^{-2} & a^{-3} & a^{-4} \\
1 & a^{-2} & a^{-4} & a^{-6} & a^{-8} \\
1 & a^{-3} & a^{-6} & a^{-9} & a^{-12} \\
1 & a^{-4} & a^{-8} & a^{-12} & a^{-16}
\end{array}\right]
$$

sendo a constante complexa $a$ definida como:

$$
a=e^{j \frac{2 \cdot \pi}{m_{s}}}=e^{j \frac{2 \cdot \pi}{5}}
$$

A matriz de transformação utilizada nas equações do rotor da máquina (9) dependerá do número de fases que constituem o rotor da máquina. Neste artigo será considerado que a máquina possui um rotor com 15 fases, sendo a matriz de transformação composta pelos autovetores da matriz $[L]^{r r}$, ou seja

$$
[Q]=\frac{1}{\sqrt{15}} \cdot\left[\begin{array}{cccccc}
1 & 1 & 1 & 1 & \cdots & 1 \\
1 & b^{-1} & b^{-2} & b^{-3} & \cdots & a^{-14} \\
1 & b^{-2} & b^{-4} & b^{-6} & \cdots & b^{-28} \\
1 & b^{-3} & b^{-6} & b^{-9} & \cdots & b^{-42} \\
\vdots & \vdots & \vdots & \vdots & \ddots & \vdots \\
1 & b^{-14} & b^{-28} & b^{-42} & \cdots & b^{-225}
\end{array}\right]
$$

sendo a constante complexa $b$ definida como segue:

$$
b=e^{j \frac{2 \cdot \pi}{m_{r}}}=e^{j \frac{2 \cdot \pi}{15}}
$$

A aplicação das transformações apresentadas em (7) e (9) no conjunto de equações que descrevem a dinâmica da parte elétrica da máquina de indução pentafásica, descritas em (1) e (2), resultam em

$$
[\widetilde{v}]^{s}=[\widetilde{R}]^{s} \cdot[\widetilde{i}]^{s}+[\widetilde{L}]^{s s} \cdot \frac{d[\widetilde{i}]^{s}}{d t}+\frac{d\left\{[\widetilde{L}]^{s r} \cdot[\widetilde{i}]^{r}\right\}}{d t}
$$




$$
[0]=[\widetilde{R}]^{r} \cdot[\widetilde{i}]^{r}+[\widetilde{L}]^{r r} \cdot \frac{d[\widetilde{i}]^{r}}{d t}+\frac{d\left\{[\widetilde{L}]^{r s} \cdot[\widetilde{i}]^{s}\right\}}{d t}
$$

onde

$$
\begin{gathered}
{[\widetilde{R}]^{s}=[O]^{-1} \cdot[R]^{s} \cdot[O]} \\
{[\widetilde{L}]^{s s}=[O]^{-1} \cdot[L]^{s s} \cdot[O]} \\
{[\widetilde{L}]^{s r}=[O]^{-1} \cdot[L]^{s r} \cdot[Q]} \\
{[\widetilde{R}]^{r}=[Q]^{-1} \cdot[R]^{r} \cdot[Q]} \\
{[\widetilde{L}]^{r r}=[Q]^{-1} \cdot[L]^{r r} \cdot[Q]} \\
{[\widetilde{L}]^{r s}=[Q]^{-1} \cdot[L]^{r s} \cdot[O]=\left\{[\widetilde{L}]^{s r}\right\}^{T *}}
\end{gathered}
$$

As mesmas transformações são aplicadas na descrição do comportamento dinâmico da parte mecânica da máquina, representando-a como função das componentes simétricas de valor instantâneo. A partir de (3), obtém-se a seguinte expressão para o torque em termos das variáveis transformadas:

$$
\begin{aligned}
T_{e}= & \frac{p}{2} \cdot[\widetilde{i}]^{r T *} \cdot \frac{d[\widetilde{L}]^{r s}}{d \phi} \cdot[\widetilde{i}]^{s}+ \\
& \frac{p}{2} \cdot[\widetilde{i}]^{s T *} \cdot \frac{d[\widetilde{L}]^{s r}}{d \phi} \cdot[\widetilde{i}]^{r}
\end{aligned}
$$

As equações (11) e (12) podem ser reescritas apenas com as componentes simétricas de valor instantâneo do harmônico fundamental e do terceiro harmônico, na forma apresentada em (20-21) e (23-24).

$$
\begin{aligned}
& \widetilde{v}_{1}^{s}=\widetilde{R}^{s} \cdot \widetilde{i}_{1}^{s}+\widetilde{L}_{1}^{s} \cdot \frac{\widetilde{d i_{1}^{s}}}{d t}+M_{1} \cdot \frac{d}{d t}\left\{e^{j \cdot \phi} \cdot \widetilde{i}_{1}^{r}\right\} \\
& 0=\widetilde{R}_{1}^{r} \cdot \widetilde{i}_{1}^{r}+\widetilde{L}_{1}^{r} \cdot \frac{\widetilde{d i}_{1}^{r}}{d t}+M_{1} \cdot \frac{d}{d t}\left\{e^{-j \cdot \phi} \cdot \widetilde{i}_{1}^{s}\right\}
\end{aligned}
$$

$$
M_{1}=\frac{\sqrt{75}}{2} \cdot \widehat{L}_{1}^{r s}
$$

Da mesma forma, observa-se que o terceiro harmônico não exerce influência nas equações para a fundamental, sendo a equação portanto completamente independente. Para o terceiro harmônico de campo, semelhante ao conjunto de equações da fundamental, obtém-se as seguintes relações:

$$
\begin{gathered}
\widetilde{v}_{3}^{s}=\widetilde{R}^{s} \cdot \widetilde{i}_{3}^{s}+\widetilde{L}_{2}^{s} \cdot \frac{\widetilde{d i}_{3}^{s}}{d t}+M_{3} \cdot \frac{d}{d t}\left\{e^{j \cdot 3 \cdot \phi} \cdot \widetilde{i}_{3}^{r}\right\} \\
0=\widetilde{R}_{3}^{r} \cdot \widetilde{i}_{3}^{r}+\widetilde{L}_{3}^{r} \cdot \frac{\widetilde{d i_{3}^{r}}}{d t}+M_{3} \cdot \frac{d}{d t}\left\{e^{-j \cdot 3 \cdot \phi} \cdot \widetilde{i}_{3}^{s}\right\} \\
M_{3}=\frac{\sqrt{75}}{2} \cdot \widehat{L}_{3}^{r s}
\end{gathered}
$$

Com base nas equações anteriores é obtido o conjunto de equações da máquina de indução pentafásica em coordenadas $q d$, considerando-se o referencial do estator. Tal transformação é obtida utilizando-se a seguinte relação, sendo $n=1,3$ :

$$
\widetilde{i}_{n}^{r s}=e^{j n \cdot \phi} \cdot \widetilde{i}_{n}^{r}
$$

As equações (27) e (28) descrevem o comportamento do motor para a componente fundamental:

$$
\begin{gathered}
{\left[\widetilde{v}_{1}\right]_{q d}^{s}=\widetilde{R}^{s} \cdot\left[\widetilde{i}_{1}\right]_{q d}^{s}+\widetilde{L}_{1}^{s} \cdot \frac{d}{d t}\left[\widetilde{i}_{1}\right]_{q d}^{s}+M_{1} \cdot \frac{d}{d t}\left[\widetilde{i}_{1}\right]_{q d}^{r s}} \\
0=\widetilde{R}_{1}^{r} \cdot\left[\widetilde{i}_{1}\right]_{q d}^{r s}+\widetilde{L}_{1}^{r} \cdot \frac{d}{d t}\left[\widetilde{i}_{1}\right]_{q d}^{r s}+ \\
M_{1} \cdot \frac{d}{d t}\left[\widetilde{i}_{1}\right]_{q d}^{s}+\omega_{e} \cdot\left[\widetilde{\Psi}_{1}\right]_{q d}^{r s}
\end{gathered}
$$

sendo

$$
\begin{aligned}
& {\left[\widetilde{v}_{1}\right]_{q d}^{s}=\left[\begin{array}{ll}
v_{1}^{s q} & v_{1}^{s d}
\end{array}\right]^{T}} \\
& {\left[\widetilde{i}_{1}\right]_{q d}^{s}=\left[\begin{array}{ll}
i_{1}^{s q} & i_{1}^{s d}
\end{array}\right]^{T}} \\
& {\left[\widetilde{i}_{1}\right]_{q d}^{r s}=\left[\begin{array}{ll}
i_{1}^{r s q} & i_{1}^{r s d}
\end{array}\right]^{T}} \\
& {\left[\widetilde{\Psi}_{1}\right]_{q d}^{r s}=\left[\begin{array}{ll}
\Psi_{1}^{r s q} & -\Psi_{1}^{r s d}
\end{array}\right]^{T}}
\end{aligned}
$$


De forma semelhante, as equações (29) e (30) são consideradas para o terceiro harmônico:

$$
\left[\widetilde{v}_{3}\right]_{q d}^{s}=\widetilde{R}^{s} \cdot\left[\widetilde{i}_{3}\right]_{q d}^{s}+\widetilde{L}_{2}^{s} \cdot \frac{d}{d t}\left[\widetilde{i}_{3}\right]_{q d}^{s}+M_{3} \cdot \frac{d}{d t}\left[\widetilde{i}_{3}\right]_{q d}^{r s}
$$

$$
\begin{aligned}
0= & \widetilde{R}_{3}^{r} \cdot\left[\widetilde{i}_{3}\right]_{q d}^{r s}+\widetilde{L}_{3}^{r} \cdot \frac{d}{d t}\left[\widetilde{i}_{3}\right]_{q d}^{r s}+ \\
& M_{3} \cdot \frac{d}{d t}\left[\widetilde{i}_{3}\right]_{q d}^{s}+3 \cdot \omega_{e} \cdot\left[\widetilde{\Psi}_{3}\right]_{q d}^{r s}
\end{aligned}
$$

sendo

$$
\begin{aligned}
& {\left[\widetilde{v}_{3}\right]_{q d}^{s}=\left[\begin{array}{ll}
v_{3}^{s q} & v_{3}^{s d}
\end{array}\right]^{T}} \\
& {\left[\widetilde{i}_{3}\right]_{q d}^{s}=\left[\begin{array}{ll}
i_{3}^{s q} & i_{3}^{s d}
\end{array}\right]^{T}} \\
& {\left[\widetilde{i}_{3}\right]_{q d}^{r s}=\left[\begin{array}{ll}
i_{3}^{r s q} & i_{3}^{r s d}
\end{array}\right]^{T}} \\
& {\left[\widetilde{\Psi}_{3}\right]_{q d}^{r s}=\left[\begin{array}{ll}
\Psi_{3}^{r s q} & -\Psi_{3}^{r s d}
\end{array}\right]^{T}}
\end{aligned}
$$

A equação do torque eletromagnético da máquina pode ser reescrita em termos das componentes qd descritas pelas equações (27) a (30).

$$
\begin{aligned}
T_{e}= & 2 \cdot p \cdot M_{1} \cdot\left[i_{1}^{s q} \cdot i_{1}^{r d}-i_{1}^{s d} \cdot i_{1}^{r q}\right]+ \\
& 6 \cdot p \cdot M_{3} \cdot\left[i_{3}^{s q} \cdot i_{3}^{r d}-i_{3}^{s d} \cdot i_{3}^{r q}\right]
\end{aligned}
$$

As equações (32) e (33) descrevem o comportamento dinâmico da parte mecânica da máquina.

$$
\begin{gathered}
-\frac{d \phi}{d t}=\omega_{e}=p \cdot \omega_{m} \\
T_{e}-T_{l}=\frac{1}{p} \cdot\left(J_{m} \cdot \frac{d^{2} \phi}{d t^{2}}+B_{m} \cdot \frac{d \phi}{d t}\right)
\end{gathered}
$$

Com base nas equações (27) a (30) que descrevem o comportamento dinâmico da parte elétrica da máquina para os componentes fundamental e terceiro harmônico, pode-se generalizar a representação na forma de espaço de estados para as componentes harmônicas de ordem $n$ :

$$
\left\{\begin{array}{l}
{[\dot{x}(t)]_{n}=\left[A\left(\omega_{e}\right)\right]_{n}[x(t)]_{n}+[B]_{n}[u(t)]_{n}} \\
{[y(t)]_{n}=[C]_{n}[x(t)]_{n}}
\end{array}\right.
$$

sendo:

$$
\begin{gathered}
{[x(t)]_{n}=\left[i_{n}^{s q} i_{n}^{s d} \Psi_{n}^{r q} \Psi_{n}^{r d}\right]^{T}} \\
{[u(t)]_{n}=\left[v_{n}^{s q} v_{n}^{s d}\right]^{T}} \\
{\left[A\left(\omega_{e}\right)\right]_{n}=\left[\begin{array}{cccc}
a_{1 n} & 0 & a_{2 n} & a_{3 n} \cdot n \cdot \omega_{e} \\
0 & a_{1 n} & -a_{3 n} \cdot n \cdot \omega_{e} & a_{2 n} \\
a_{4 n} & 0 & a_{5 n} & -n \cdot \omega_{e} \\
0 & a_{4 n} & n \cdot \omega_{e} & a_{5 n}
\end{array}\right]}
\end{gathered}
$$

$$
\begin{gathered}
{[B]_{n}=\left[\begin{array}{cc}
b_{1 n} & 0 \\
0 & b_{1 n} \\
0 & 0 \\
0 & 0
\end{array}\right]} \\
{[C]_{y n}=\left[\begin{array}{llll}
1 & 0 & 0 & 0 \\
0 & 1 & 0 & 0
\end{array}\right]}
\end{gathered}
$$

definindo as constantes:

$$
\begin{aligned}
a_{1 n} & =-\left(\frac{\widetilde{R}^{s} \cdot \widetilde{L}_{n}^{r}+M_{n}^{2} \cdot \tau_{r n}}{K_{n}}\right) \\
a_{2 n} & =\frac{M_{n} \cdot \tau_{r n}}{K_{n}} \\
a_{3 n} & =\frac{M_{n}}{K_{n}} \\
a_{4 n} & =M_{n} \cdot \tau_{r n} \\
a_{5 n} & =-\tau_{r n} \\
b_{1 n} & =\frac{\widetilde{L}_{n}^{r}}{K_{n}} \\
K_{n} & =\widetilde{L}_{n}^{s} \widetilde{L}_{n}^{r}-M_{n}^{2} \\
\tau_{r n} & =\frac{\widetilde{R}_{n}^{r}}{\widetilde{L}_{n}^{r}}
\end{aligned}
$$

Observa-se que o modelo representado na forma de espaço de estados utiliza as correntes do estator e os fluxos do rotor como variáveis, sendo utilizada a relação $\widetilde{\psi}_{n}^{r s}=\widetilde{L}_{n}^{r \widetilde{i}_{n}^{r s}+}$ $M_{n} \widetilde{i}_{n}^{s}$ para obtenção dos fluxos do rotor. Tal transformação de variáveis se faz necessária para o equacionamento do observador de fluxos do rotor apresentado na seção seguinte deste trabalho. 


\section{OBSERVADOR ROBUSTO PARA MÁ- QUINAS PENTAFÁSICAS}

Nesta seção será descrita a forma de adequação do modelo do motor de indução pentafásico utilizado para síntese do observador robusto. Primeiramente será assumido que a velocidade do rotor e que as correntes do estator são variáveis disponíveis para medida.

Considerando o modelo da máquina apresentada na equação (34), sendo $x_{n}(t)$ o vetor de estados, $u_{n}(t)$ o vetor de entradas e $y_{n}(t)$ o vetor de saídas, composto pelas correntes do estator da máquina. Para efeito de projeto do observador será acrescido ao modelo um vetor de saídas adicionais $z_{n}(t)$, que representam os fluxos do rotor e que são os estados a serem estimados, de forma que o modelo a ser considerado para o projeto do observador de estados é apresentado em (40).

$$
\left\{\begin{array}{l}
{[\dot{x}(t)]_{n}=\left[A\left(\omega_{e}\right)\right]_{n}[x(t)]_{n}+[B]_{n}[u(t)]_{n}} \\
{[y(t)]_{n}=\left[C_{y}\right]_{n}[x(t)]_{n}} \\
{[z(t)]_{n}=\left[C_{z}\right]_{n}[x(t)]_{n}}
\end{array}\right.
$$

sendo

$$
\left[C_{z}\right]_{n}=\left[\begin{array}{cccc}
0 & 0 & 1 & 0 \\
0 & 0 & 0 & 1
\end{array}\right]
$$

Observa-se que o modelo (34) é parametrizado em relação a velocidade elétrica, $\omega_{e}$, da máquina. Supondo que a velocidade é medida online e possui valores máximos e mínimos conhecidos, o sistema (34) pode ser visto como um sistema linear a parâmetros variantes, ou LPV do inglês Linear Parameter Varying (Leith e Leithead, 2000), onde $\omega_{e}$ é a variável de escalonamento. Além disso, frequentemente, a resistência do rotor é modelada como um parâmetro incerto variante no tempo, com valores máximos e mínimos conhecidos, visando considerar no modelo da máquina as variações provocadas tipicamente pelo aquecimento do rotor (Chan e Wang, 1990).

Neste trabalho, assume-se que a velocidade elétrica satisfaz a seguinte relação:

$$
\omega_{e} \in \mathcal{W}:=\left\{\omega_{e}:\left|\omega_{e}\right| \leq \bar{\omega}\right\}
$$

e considera-se que a resistência própria de uma fase do rotor $R_{r}$ é uma função do tempo composta pela resistência do anel e pela resistência da barra, isto é:

$$
R_{r}=2\left(R_{a}^{r}+R_{b}^{r}\right),
$$

onde a resistência do anel, $R_{a}^{r}$, é considerada fixa e a resistência da barra, $R_{b}^{r}$, é modelada como uma variável incerta com faixa de variação conhecida, isto é: $R_{b}^{r} \in[\underline{R}, \bar{R}]$. Por conveniência, a resistência da barra será reescrita na seguinte forma:

$$
R_{b}^{r}=R_{b}^{r 1}+R_{b}^{r 2} \delta, \delta \in \Delta:=[-1,1]
$$

onde $\delta=\delta(t)$ é um parâmetro incerto variante no tempo, $\Delta$ representa o conjunto de valores admissíveis de $\delta, R_{b}^{r 1}$ é o valor nominal da resistência da barra e $R_{b}^{r 2}$ o desvio máximo em relação ao valor nominal. Considerando os limites de variação de $R_{b}^{r}$ definidos anteriormente, obtém-se as seguintes relações

$$
R_{b}^{r 1}=\frac{\bar{R}+\underline{R}}{2} \text { e } R_{b}^{r 2}=\frac{\bar{R}-\underline{R}}{2} \text {. }
$$

Na definição do modelo da máquina aparece a resistência transformada $\widetilde{R}_{n}^{r}$ que assume os seguintes valores, como proposto em (Pereira et al., 2006) para o primeiro e terceiro harmônicos:

$$
\widetilde{R}_{n}^{r}=2 R_{a}^{r}+4 R_{b}^{r} \sin ^{2}\left(\frac{n \cdot \varepsilon}{2}\right)
$$

onde $\varepsilon=2 \pi / 15$ é o ângulo elétrico entre duas barras vizinhas do rotor.

Levando em consideração a equação (43), a resistência transformada $\widetilde{R}_{n}^{r}$ é redefinida da seguinte maneira:

$$
\widetilde{R}_{n}^{r}=\widetilde{R}_{n 1}^{r}+\widetilde{R}_{n 2}^{r} \delta, \delta \in \Delta,
$$

onde

$$
\begin{aligned}
\widetilde{R}_{n 1}^{r} & =2 R_{a}^{r}+4 R_{b}^{r 1} \cdot \sin ^{2}\left(\frac{n \cdot \varepsilon}{2}\right) \\
\widetilde{R}_{n 2}^{r} & =4 R_{b}^{r 2} \cdot \sin ^{2}\left(\frac{n \cdot \varepsilon}{2}\right)
\end{aligned}
$$

Em resumo, a matriz $A_{n}\left(\omega_{e}\right)$ que define a dinâmica da máquina depende de dois parâmetros variantes no tempo $\omega_{e}$ e $\delta$, que representam respectivamente a velocidade elétrica (suposta mensurável e disponível online) e a incerteza presente no valor da resistência do rotor (com valores máximo e mínimo conhecidos).

Visando o projeto do observador, a matriz $\left[A\left(\omega_{e}\right)\right]_{n}$ da equação (37), doravante representada por $A_{n}\left(\omega_{e}\right)$, é decomposta em duas partes como definido a seguir:

$$
A_{n}\left(\omega_{e}\right)=A_{n}\left(\omega_{e}, \delta\right)=A_{n \omega}\left(\omega_{e}\right)+A_{n r}(\delta)
$$

onde $A_{n \omega}\left(\omega_{e}\right)$ é uma matriz afim em $\omega_{e}$ e $A_{n r}(\delta)$ uma matriz linear em $\delta$ que serão definidas em seguida.

Os elementos $a_{i n}$ da matriz $A_{n}\left(\omega_{e}, \delta\right)$, definida em (47), que dependem da incerteza $\delta$, podem ser reescritos na seguinte forma:

$$
a_{i n}=a_{i n}(\delta)=\bar{a}_{i n}+\widetilde{a}_{i n} \delta, i=1,3,4,5
$$


onde $\bar{a}_{i n}$ corresponde ao valor nominal de $a_{i n}(\delta)$ e $\widetilde{a}_{i n}$ a magnitude da incerteza. Com base na definição acima, as matrizes $A_{n \omega}\left(\omega_{e}\right)$ e $A_{n r}(\delta)$ são definidas como:

$$
\begin{aligned}
A_{n \omega}\left(\omega_{e}\right) & =\left[\begin{array}{cccc}
\bar{a}_{1 n} & 0 & \bar{a}_{2 n} & a_{3 n} n \omega_{e} \\
0 & \bar{a}_{1 n} & -a_{3 n} n \omega_{e} & \bar{a}_{2 n} \\
\bar{a}_{4 n} & 0 & \bar{a}_{5 n} & -n \omega_{e} \\
0 & \bar{a}_{4 n} & n \omega_{e} & \bar{a}_{5 n}
\end{array}\right] \\
A_{n r}(\delta) & =\left[\begin{array}{cccc}
\widetilde{a}_{1 n} \delta & 0 & \widetilde{a}_{2 n} \delta & 0 \\
0 & \widetilde{a}_{1 n} \delta & 0 & \widetilde{a}_{2 n} \delta \\
\widetilde{a}_{4 n} \delta & 0 & \widetilde{a}_{5 n} \delta & 0 \\
0 & \widetilde{a}_{4 n} \delta & 0 & \widetilde{a}_{5 n} \delta
\end{array}\right]
\end{aligned}
$$

onde os valores de $\bar{a}_{i n}$ e $\widetilde{a}_{i n}$ são obtidos a partir das definições (37) e (45) levando em consideração a relação (48).

Com o objetivo de estimar as componentes harmônicas do fluxo do rotor, representadas pelo sinal $z_{n}(t)$, propõe-se o seguinte observador de estados baseado no observador clássico de Luenberger:

$$
\left\{\begin{array}{l}
\dot{\hat{x}}_{n}(t)=A_{n \omega}\left(\omega_{e}\right) \hat{x}_{n}(t)+B_{n} u_{n}(t)+L_{n}\left(\omega_{e}\right) e_{y n} \\
\hat{y}_{n}(t)=C_{y n} \hat{x}(t) \\
\hat{z}_{n}(t)=C_{z n} \hat{x}(t) \\
e_{y n}(t)=y_{n}(t)-\hat{y}_{n}(t)
\end{array}\right.
$$

onde $\hat{x}_{n} \in \mathbb{R}^{4}$ é o vetor de estados do observador; $\hat{y}_{n} \in \mathbb{R}^{2}$ é a estimativa das variáveis de saída do modelo (correntes do estator) e $\hat{z}_{n} \in \mathbb{R}^{2}$ é a estimativa dos fluxos do rotor; $L_{n}\left(\omega_{e}\right)$ é uma função matricial em $\omega_{e}$ a ser determinada; e $A_{n \omega}\left(\omega_{e}\right)$ é a matriz definida em (49).

Neste trabalho, a matriz de ganhos do observador $L_{n}\left(\omega_{e}\right)$ é uma função afim em $\omega_{e}$, isto é:

$$
L_{n}\left(\omega_{e}\right)=L_{0 n}+L_{1 n} \omega_{e}
$$

onde $L_{0 n}$ e $L_{1 n}$ são matrizes constantes.

Seja $e_{n}(t):=x_{n}(t)-\hat{x}_{n}(t)$ o vetor de estados do seguinte sistema dinâmico:

$$
\left\{\begin{aligned}
\dot{e}_{n}(t) & =\left(A_{n \omega}-L_{n} C_{y n}\right) e_{n}(t)+A_{n r}(\delta) x_{n}(t) \\
z_{n e}(t) & =C_{z n} e_{n}(t) \\
y_{n e}(t) & =C_{y n} e_{n}(t)
\end{aligned}\right.
$$

onde $A_{n \omega}=A_{n \omega}\left(\omega_{e}\right), L_{n}=L_{n}\left(\omega_{e}\right), z_{n e}(t):=z_{n}(t)-$ $\hat{z}_{n}(t)$ e $y_{n e}(t):=y_{n}(t)-\hat{y}_{n}(t)$.

Neste trabalho, o sinal $A_{n r}(\delta) x_{n}(t)$ (que aparece na dinâmica do erro devido à incerteza do modelo da máquina) será modelado como uma perturbação externa à dinâmica do sistema (52).

Por conveniência, são definidas as seguintes variáveis auxiliares:

$$
\begin{gathered}
B_{n e}(\delta)=A_{n r}(\delta), \\
A_{n e}\left(\omega_{e}\right)=A_{n \omega}\left(\omega_{e}\right)-L_{n}\left(\omega_{e}\right) C_{y n} .
\end{gathered}
$$

Com as definições acima, o sistema que descreve a dinâmica do erro dos estados observados pode ser reescrito na seguinte forma compacta:

$$
\left\{\begin{aligned}
\dot{e}_{n}(t) & =A_{n e}\left(\omega_{e}\right) e_{n}(t)+B_{n e}(\delta) x_{n}(t) \\
z_{n e}(t) & =C_{z n} e_{n}(t) \\
y_{n e}(t) & =C_{y n} e_{n}(t)
\end{aligned}\right.
$$

Para o projeto da matriz $L_{n}\left(\omega_{e}\right)$, utiliza-se o conceito da norma $H_{\infty}$, na qual busca-se uma solução que minimize o efeito da perturbação $x_{n}(t)$ no erro de estimação $z_{n e}(t)$ para todo $\delta \in \Delta$. Com este objetivo, considere a seguinte definição para o o ganho- $\mathcal{L}_{2}$ de $x_{n}(t)$ para $z_{n e}(t)$ :

$$
\left\|G_{x z}\right\|_{\infty}=\sup _{\delta \in \Delta, w \neq 0} \frac{\left\|z_{n e}(t)\right\|_{2}}{\left\|x_{n}(t)\right\|_{2}}
$$

Um limitante superior $\gamma$ para $\left\|G_{p z}\right\|_{\infty}$ pode ser obtido através da seguinte relação:

$$
\dot{V}(e)+\frac{1}{\gamma} z_{n e}(t)^{T} z_{n e}(t)-\gamma x_{n}(t)^{T} x_{n}(t)<0
$$

onde $V(e)>0$ é uma função de Lyapunov para o sistema (54). Supondo condições iniciais nulas e integrando a desigualdade em (55) de 0 a $+\infty$ obtém-se:

$$
\left\|z_{n e}(t)\right\|_{2} \leq \gamma\left\|x_{n}(t)\right\|_{2}
$$

Neste trabalho, utiliza-se uma função de Lyapunov quadrática, isto é

$$
V_{n}=e^{T} P_{n} e
$$

sendo $P_{n} \in \mathbb{R}^{4 \times 4}$ uma matriz simétrica e positiva definida. Considerando (54), (55) e (56), obtém-se a seguinte desigualdade:



onde $n=1,3, A_{n e}=A_{n e}\left(\omega_{e}\right)$ e $B_{n e}=B_{n e}(\delta)$.

Definindo $M_{n}\left(\omega_{e}\right)=P_{n} L_{n}\left(\omega_{e}\right)$ e aplicando-se o teorema de Schur na matriz da desigualdade acima, chega-se ao seguinte problema de otimização LMI:

$$
\min \gamma_{n}:\left\{\begin{array}{l}
P_{n}=P_{n}^{T}>0 \\
{\left[\begin{array}{ccc}
\Pi_{n} & P_{n} B_{n e} & C_{z n}^{T} \\
B_{n e}^{T} P_{n} & -\gamma_{n} I_{4} & 0 \\
C_{z n} & 0 & -\gamma_{n} I_{2}
\end{array}\right]<0} \\
\forall\left(\omega_{e}, \delta\right) \in \mathcal{V}(\mathcal{W} \times \Delta)
\end{array}\right.
$$


Tabela 1: Parâmetros da máquina.

\begin{tabular}{|c|c|}
\hline Parâmetro & Valor \\
\hline$\widetilde{\mathrm{R}}^{\mathrm{s}}$ & $3,9 \Omega$ \\
\hline$\widetilde{\mathrm{R}}_{1}^{\mathrm{r}}$ & $10,5 \mu \Omega$ \\
\hline$\widetilde{\mathrm{R}}_{3}^{\mathrm{r}}$ & $55,2 \mu \Omega$ \\
\hline$\widetilde{\mathrm{L}}_{1}^{\mathrm{s}}$ & $168,9 \mathrm{mH}$ \\
\hline$\widetilde{\mathrm{L}}_{2}^{\mathrm{s}}$ & $21,0 \mathrm{mH}$ \\
\hline$\widetilde{\mathrm{L}}_{1}^{\mathrm{r}}$ & $0,71 \mu \mathrm{H}$ \\
\hline$\widetilde{\mathrm{L}}_{3}^{\mathrm{r}}$ & $0,92 \mu \mathrm{H}$ \\
\hline$\widehat{\mathrm{L}}_{1}^{\mathrm{rs}}$ & $76,4 \mu \mathrm{H}$ \\
\hline$\widehat{\mathrm{L}}_{3}^{\mathrm{rs}}$ & $21,5 \mu \mathrm{H}$ \\
\hline $\mathrm{B}_{\mathrm{m}}$ & $5,9.10^{-4} \mathrm{~N} \cdot \mathrm{m} / \mathrm{rad} . \mathrm{s}^{-1}$ \\
\hline $\mathrm{J}_{\mathrm{m}}$ & $7,8.10^{-3} \mathrm{~kg} / \mathrm{m}^{2}$ \\
\hline
\end{tabular}

onde $n=1,3 ; B_{n e}=B_{n e}(\delta) ; P_{n} \in \mathbb{R}^{4 \times 4}, M_{0 n}, M_{1 n} \in$ $\mathbb{R}^{4 \times 2}$ e $\gamma_{n} \in \mathbb{R}$ são as variáveis de decisão; $\mathcal{V}(\mathcal{W} \times \Delta)$ representa o conjunto de vertices do politopo obtido pelo produto cartesiano entre $\mathcal{W}$ e $\Delta ; \mathrm{e}$

$$
\Pi_{n}=P_{n} A_{n \omega}+A_{n \omega}^{T} P_{n}-M_{n} C_{y n}-C_{y n}^{T} M_{n}^{T},
$$

sendo $A_{n \omega}=A_{n \omega}\left(\omega_{e}\right)$ e $M_{n}=M_{0 n}+M_{1 n} \omega_{e}$.

Se existir uma solução factível para o problema de otimização definido em (58), então a matriz de ganhos do observador é dada por:

$$
L_{n}\left(\omega_{e}\right)=P_{n}^{-1}\left(M_{0 n}+M_{1 n} \omega_{e}\right) .
$$

\section{RESULTADOS DE SIMULAÇÃO}

Com base no modelo matemático da máquina de indução pentafásica, foi desenvolvido um ambiente de simulação em Matlab/Simulink ${ }^{\circledR}$. As equações que descrevem o comportamento da máquina foram inseridas neste ambiente, juntamente com os demais componentes que compõem o acionamento da máquina (inversor, filtros anti-aliasing, controlador de corrente por histerese, etc), empregando a plataforma de simulação descrita em (Spiller et al., 2002). Os parâmetros considerados foram obtidos com base no protótipo de uma máquina pentafásica, cujos parâmetros são dados na Tabela 1 .

Para validação do observador robusto proposto neste trabalho foi empregado o esquema de controle por orientação direta do campo. Através do modelo desacoplado proposto anteriormente, é possível obter uma estratégia de controle que permite a orientação independente dos componentes do harmô- nico fundamental e do terceiro harmônico. A Figura 1 apresenta um diagrama de blocos da proposta de controle de motores pentafásicos com a inclusão de harmônicos da indução no entreferro.

Utiliza-se a proposição padrão de orientação direta do campo, realizada no referencial síncrono, empregando-se o observador proposto para obtenção dos fluxos das componentes do primeiro e do terceiro harmônicos do rotor. Os fluxos observados são comparados com os fluxos de referência, sendo os erros de fluxo referentes ao primeiro e terceiro harmônicos utilizados como variável de entrada de dois controladores do tipo proporcional integral que irão determinar as componentes de correntes diretas, relativas ao primeiro e terceiro harmônicos, também no referencial síncrono. O mesmo esquema é realizado para a obtenção das componentes de correntes de quadratura, empregando neste caso a informação do erro de velocidade como entrada dos controladores. Uma vez determinada as correntes de primeiro e terceiro harmônicos nos eixos direto e quadratura, todas elas relacionadas ao referencial síncrono da máquina, é realizada a transformação de coordenadas para o referencial do estator.

Conforme observado, para a orientação direta do campo é necessária a informação dos fluxos do rotor da máquina de indução. Estes fluxos são obtidos através do observador robusto descrito na seção anterior, cujas matrizes de ganhos são apresentadas a seguir:

$$
\begin{aligned}
& L_{01}=\left[\begin{array}{cc}
5,2057 & 0,0000 \\
0,0000 & 5,2057 \\
-0,0002 & -0,0000 \\
-0,0000 & -0,0002
\end{array}\right] \times 10^{6} \\
& L_{11}=\left[\begin{array}{cc}
-0,0018 & 0,5544 \\
-0,5543 & 0,0009 \\
-0,0001 & -2,7771 \\
2,7771 & -0,0001
\end{array}\right] \\
& L_{03}=\left[\begin{array}{cc}
6,5248 & 0,0000 \\
0,0000 & 6,5248 \\
-0,0001 & -0,0000 \\
-0,0000 & -0,0001
\end{array}\right] \times 10^{6} \\
& L_{13}=\left[\begin{array}{cc}
0,0138 & 0,3615 \\
-0,3608 & 0,0080 \\
-0,0088 & -15,505 \\
15,498 & 0,0088
\end{array}\right]
\end{aligned}
$$

Inicialmente será avaliado apenas o comportamento do observador proposto, comparando-se os resultados do controle por orientação de campo em dois sistemas equivalentes:

- Sistema com Fluxos Medidos do Rotor, no qual os fluxos do rotor são obtidos diretamente da equações dinâ- 


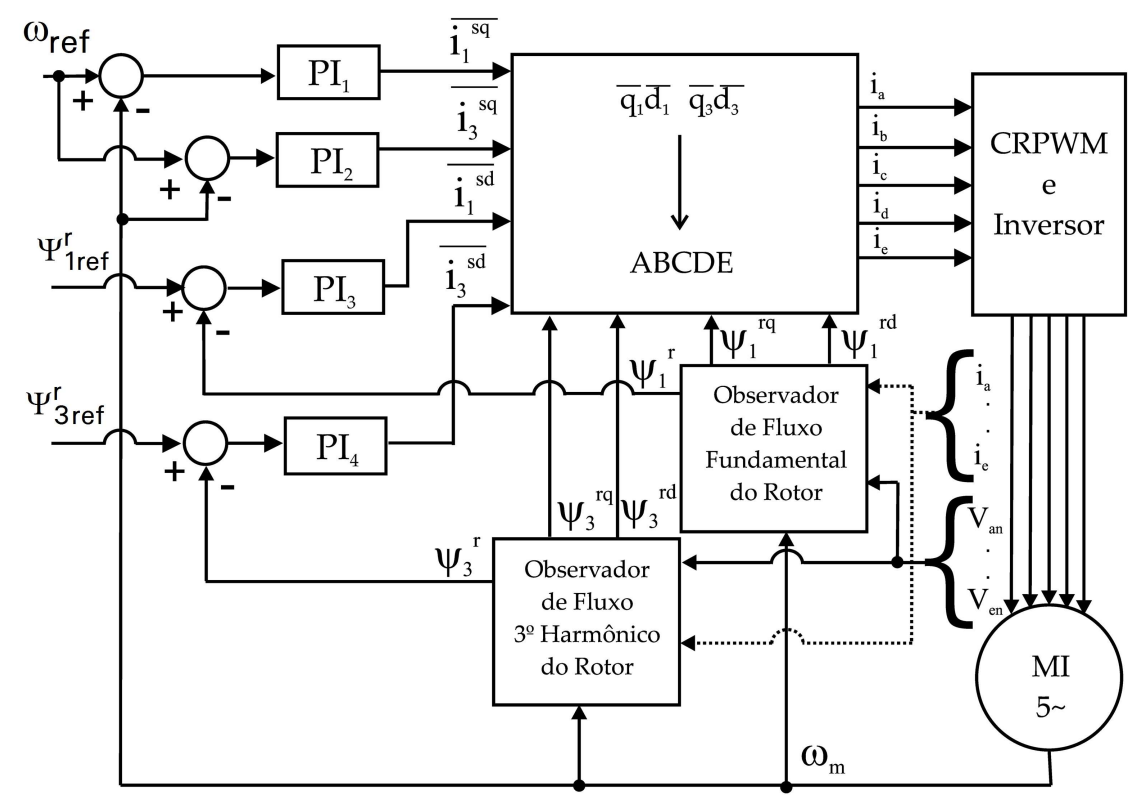

Figura 1: Diagrama de blocos da proposta de controle.

micas da máquina pentafásica. Seria o caso ideal que não é praticamente realizável. Seus fluxos RMS são nomeados $\Psi_{1}^{r m}$ e $\Psi_{3}^{r m}$ para o primeiro e terceiro harmônicos respectivamente.

- Sistema com Fluxos Observados do Rotor, no qual os fluxos do rotor são obtidos empregando o observador projetado. Seus fluxos RMS são nomeados $\Psi_{1}^{r o}$ e $\Psi_{3}^{r o}$ para o primeiro e terceiro harmônicos respectivamente.

Nas simulações a resistência da barra do rotor $R_{b r}$ é alterada linearmente até alcançar $45 \%$ além do seu valor nominal, iniciando no instante de tempo 0,5 segundos e finalizando no instante de tempo 0,7 segundos. Adicionalmente a referência de velocidade é modificada conforme apresentado na Figura 2. No instante de tempo 0,2 segundos a referência de velocidade é estabelecida em 900 rpm, e no instante de tempo 0,8 segundos a velocidade é reduzida para metade deste valor, ou seja $450 \mathrm{rpm}$.

A Figura 2 apresenta o comportamento das velocidades do rotor dos dois sistemas juntamente com o sinal de referência. Observa-se que as curvas estão praticamente sobrepostas durante todo o intervalo de simulação.

Nas figuras 3 e 4 são mostrados os erros entre os fluxos do rotor medidos e estimados, tanto para o primeiro quanto para o terceiro harmônico. Pode-se observar que no caso do primeiro harmônico os erros são máximos nos tempos onde a velocidade varia, mas logo tende a se estabilizar. Além disso

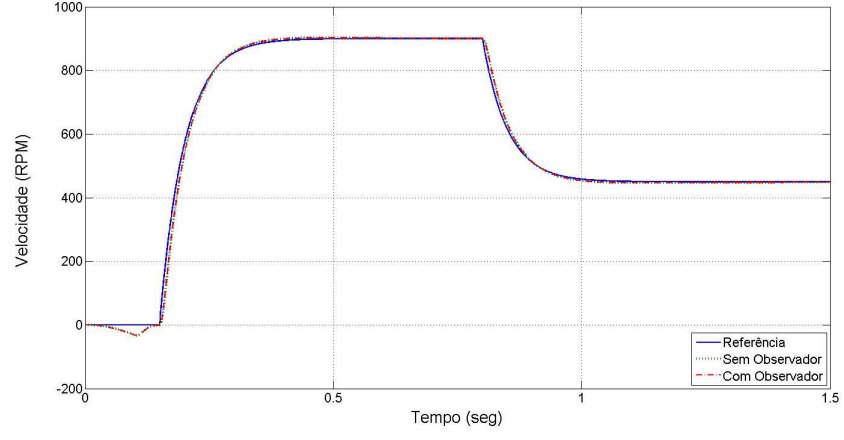

Figura 2: Comparação de Velocidades

existe uma pequena variação do erro entre os instantes 0,5 e 0,7 segundo devido à variação da resistência do rotor. $\mathrm{O}$ comportamento do erro do fluxo para o terceiro harmônico apresenta características similares às do primeiro harmônico. Observa-se também que o erro máximo para a componente de fluxo do primeiro harmônico foi de $1,2 \cdot 10^{-5} \mathrm{~Wb}$, ocorrendo em torno de 0,8 segundo, o que resulta em um erro percentual de $1,2 \%$, valor obtido em relação a referência fixa de 0,001 Wb para a componente do primeiro harmônico de fluxo do rotor. Para a componente de terceiro harmônico, o erro máximo ocorre em torno de 0,2 segundo com um valor aproximado de $-1,11 \cdot 10^{-5} \mathrm{~Wb}$, o que resulta em um erro percentual de aproximadamente $3,7 \%$, valor obtido em relação a referência fixa de $0,0003 \mathrm{~Wb}$ para a componente do terceiro harmônico de fluxo do rotor. As referências de 
fluxo das componentes fundamental e de terceiro harmônico foram selecionadas para que a máquina apresentasse comportamento dinâmico semelhante ao obtido com a orientação do campo realizada somente pelo harmônico fundamental, com seus valores numéricos apresentados na Tabela 3 . A primeira linha desta tabela refere-se ao fluxo da componente fundamental para o esquema de controle tradicional enquanto as duas últimas linhas referem-se aos fluxos da componente fundamental e do terceiro harmônico do esquema de controle proposto. Como resultado foi possível definir um valor de fluxo resultante menor para a técnica proposta, implicando correntes menores para uma mesma condição de carga.



Figura 3: Erro do Fluxo para o $1^{\circ}$ Harmônico. $\left(\left|\Psi_{1}^{r m}\right|-\left|\Psi_{1}^{r o}\right|\right)$

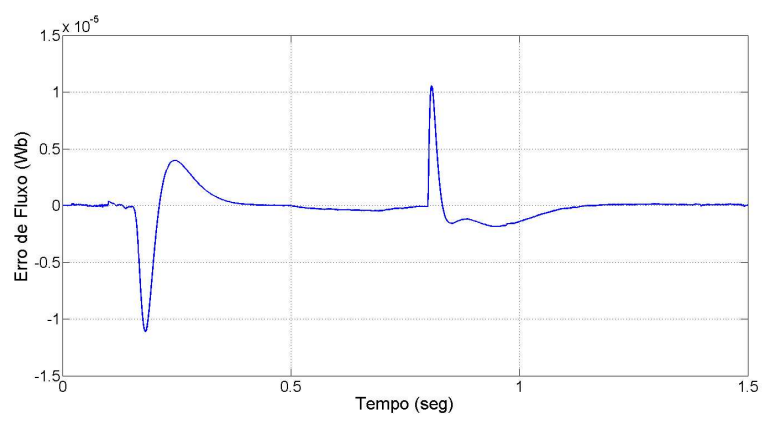

Figura 4: Erro do Fluxo para o $3^{\circ}$ Harmônico. $\left(\left|\Psi_{3}^{r m}\right|-\left|\Psi_{3}^{r o}\right|\right)$

Adicionalmente, de forma a avaliar o comportamento da máquina considerando a inclusão dos efeitos das componentes de terceiro harmônico foram realizados testes com a máquina operando com controle por orientação de campo tradicional, ou seja, quando somente os sinais do harmônico fundamental são utilizados para a orientação do campo, e com uma proposta de controle que considera a inclusão das componentes fundamental e terceiro harmônico. Os testes foram repetidos utilizando diferentes valores de carga na máquina. Os ganhos dos controladores são apresentados na Tabela 2, tendo sido estabelecidos pelo valor numérico do somatório do erro médio quadrático entre a curva de referência de velocidade e a curva de velocidade observada no rotor da máquina.

A Figura 5 apresenta um gráfico dos valores de corrente do estator em função do conjugado. Estes valores foram obtidos das simulações realizadas com os dois esquemas de controle. No gráfico pode-se verificar uma redução na corrente RMS do estator para o controle por orientação de campo com inclusão do terceiro harmônico. De acordo com as simulações, com a utilização do esquema de controle proposto é possível obter uma redução na corrente em relação a uma máquina operando no método tradicional que varia de $14 \%$ para a máquina operando com carga nominal até $17 \%$ para funcionamento a vazio.



Figura 5: Gráfico comparativo entre as estratégias de controle.

Tabela 2: Ganhos dos controladores.

\begin{tabular}{|c|c|c|c|c|}
\hline Ganho & $\overline{\mathrm{i}_{1}^{\text {sq }}}$ & $\overline{\mathrm{i}_{1}^{\text {sd }}}$ & $\overline{\mathrm{i}_{3}^{\text {sq }}}$ & $\overline{\mathrm{i}_{3}^{\text {sd }}}$ \\
\hline $\mathrm{K}_{\mathrm{p}}$ & 0,07 & $1,0.10^{4}$ & 0,21 & $1,0.10^{4}$ \\
\hline $\mathrm{K}_{\mathrm{i}}$ & 0,36 & $2,0.10^{5}$ & 0,36 & $8,0.10^{5}$ \\
\hline
\end{tabular}

Tabela 3: Referências de fluxo.

\begin{tabular}{|c|c|c|}
\hline Estratégia de Controle & Fluxo & Valor \\
\hline Fundamental & $\Psi_{1}^{\text {sd }}$ & $0,00175 \mathrm{~Wb}$ \\
\hline Fundamental e & $\Psi_{1}^{\text {sd }}$ & $0,001 \mathrm{~Wb}$ \\
\cline { 2 - 3 } Terceiro Harmônico & $\Psi_{3}^{\text {sd }}$ & $0,0003 \mathrm{~Wb}$ \\
\hline
\end{tabular}

A Figura 6 apresenta as curvas do conjugado elétrico relativas ao harmônico fundamental e ao terceiro harmônico. Verifica-se neste caso a presença de conjugado gerado pelo terceiro harmônico, o que não ocorre quando se emprega as 

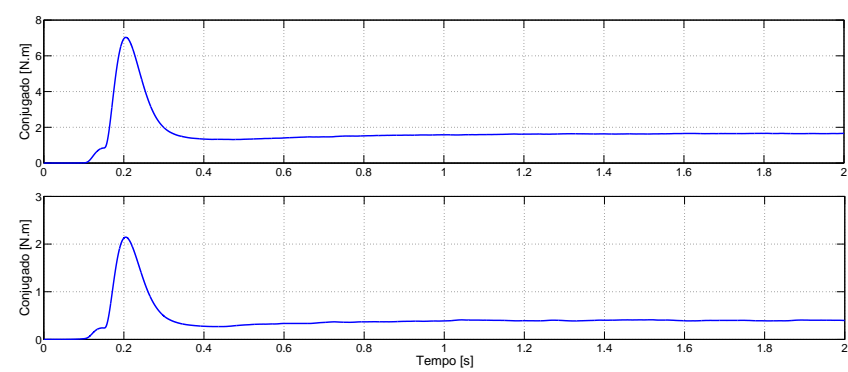

Figura 6: Conjugado elétrico da máquina operando com a metodologia de controle proposta: (a) - harmônico fundamental, (b) - terceiro harmônico.

técnicas convencionais de orientação de campo. O torque total da máquina é igual ao somatório destas curvas.

Cabe salientar que os resultados obtidos foram avaliados somente em simulação, enfocando as vantagens do esquema de controle proposto sob o ponto de vista da redução das correntes RMS da máquina. Ainda não foram analisados os efeitos práticos que podem ocorrer com relação as perdas Joule inerentes da inclusão de um componente de corrente com frequência três vezes superior a componente fundamental da máquina.

\section{CONCLUSÕES}

Apresentou-se neste artigo a proposta de um observador robusto aplicado a máquinas de indução pentafásicas, considerando a modelagem da máquina em variáveis de componentes simétricas de valor instantâneo. Com base neste modelo, obteve-se a representação das equações dinâmicas da máquina para as componentes fundamental e de terceiro harmônico desacopladas nas variáveis elétricas. A partir desta representação foi formulado um observador robusto para as componentes fundamentais e de terceiro harmônico, cujas matrizes que compõem os ganhos do observador são determinadas através de um conjunto de LMIs - Desigualdades Matriciais Lineares, garantindo características de otimalidade associadas a critérios de desempenho previamente definidos, dentro de todo o intervalo considerado para variação de velocidade e de resistência do rotor. Os resultados de simulação mostraram que os fluxos do rotor observados apresentaram um comportamento muito próximo dos fluxos rotor medidos, validando a metodologia de projeto proposta neste artigo. Utilizando os fluxos observados, foi realizado o controle por orientação direta do campo, com imposição das componentes de fluxo do primeiro e do terceiro harmônico. Os resultados obtidos foram comparados com o controle por orientação do campo tradicional, constatando-se um incremento no torque da máquina associado a inclusão das componentes de terceiro harmônico.

\section{REFERÊNCIAS}

Blaschke, F. (1972). The Principle of the Field-Orientation as Applied to the New Transvector Close-Loop System for Rotating-Field Machine, Siemens Review 39(5): 217220.

Chan, C. C. e Wang, H. (1990). An effective method for rotor resistance identification for high-performance induction motor vector control, IEEE Trans. Ind. Electron. 37(6): 477-482.

Coutinho, D. F., Curcio, M., Pereira, L. F. A. e Haffner, J. F. (2004). State Observer Design for Bilinear Systems with Application to Induction Motors, Proceedings of the 43rd IEEE Conference on Decision and Control, Vol. 1, pp. 686-691.

Coutinho, D. F., Fu, M. e Trofino, A. (2004). Robust analysis and control for a class of uncertain nonlinear discretetime systems, 53: 377-393.

Coutinho, D. F., Pereira, L. F. A., Salton, A. e Heck, G. (2007). A Robust Discrete-Time Observer for Induction Motors, Proceedings of the European Control Conference, Greece, pp. 5147-5153.

Coutinho, D. F. e Trofino, A. (2002). Análise de Sistemas Não-Lineares Incertos: uma Abordagem LMI, Controle e Automação 13(2): 94-104.

Coutinho, D. F., Trofino, A. e Fu, M. (2002). Guaranteed Cost Control of Uncertain Nonlinear Systems via Polynomial Lyapunov Functions, 47(9): 1575-1580.

Ghaoui, L. E. e Niculescu, L. I. (2000). Advances in Linear Matrix Inequality Methods in Control, SIAM.

Huang, Y. e Lu, W.-M. (1996). Nonlinear Optimal Control: Alternatives to Hamilton-Jacobi Equation, Proceedings of the 35th Conference on Decision and Control, Kobe, pp. 3942-3947.

Jacobina, C. B., Freitas, I. S., Oliveira, T. M., da Silva, E. R. C. e Lima, A. M. N. (2004). Fault tolerant control of five-phase ac motor drive, 35th Annual IEEE Power Electronics Specialist Conference.

Leith, D. e Leithead, W. (2000). Survey of gain-scheduling analysis \& design, Int J Control 73(11): 1001-1025.

Luenberger, D. G. (1966). Observers for Multivariable Systems, 11: 190-197. 
Lyra, R. e Lipo, T. (2002). Torque density improvement in a six-phase induction motor with third harmonic current injection, Industry Applications, IEEE Transactions on 38(5): 1351-1360.

Moreira, J. e Lipo, T. (1992). Modeling of saturated ac machines including air gap flux harmonic components, Industry Applications, IEEE Transactions on 28(2): 343349.

Pereira, L. A., Scharlau, C. C., Pereira, L. F. A. e Haffner, J. F. (2006). General model of a five-phase induction machine allowing for harmonics in the air gap field, IEEE Trans. Energy Conversion 21(4): 891-899.

Pereira, L. A., Scharlau, C. C., Pereira, L. F. A. e Haffner, S. L. (2010). Controle de máquinas de indução pentafásicas com otimização do campo no entreferro, Controle e Automação 1(1): 1-10.

Pereira, L. F. A., Haffner, J. F., Gründling, H. A. e Hemerly, E. M. (1998). Direct Vector Control for a Servopositioner Using an Alternative Rotor Flux Estimation Algorithm, Proceedings of the 24th Annual Conference of the IEEE Industrial Electronics Society, Vol. 3, Aachen, pp. 1603-1608.

Petersen, I. R. e Savkin, A. V. (1999). Robust Kalman Filtering for Signals and Systems with Large Uncertainties, Birkhauser.

Prempain, E. e Postlethwaite, I. (2002). $H_{\infty}$ Design for an Induction Motor, Proceedings of the 15th IFAC World Congress, Barcelona, Spain.

Salvatore, L., Stasi, S. e Tarchioni, L. (1993). A New EKFBased Algorithm for Flux Estimation in Induction Machines, 40(5): 496-504.

Scharlau, C. C., Pereira, L. F. A., Pereira, L. A. e Haffner, S. L. (2008). Performance of a five-phase induction machine with optimized air gap field under open loop VIf control, IEEE Trans. Energy Conversion 1.

Spiller, P. A., Haffner, J. F. e Pereira, L. F. A. (2002). Aplicação em tempo real de ferramentas de simulação e implementação de técnicas de controle de máquinas de indução em ambiente Matlab/Simulink, XIV Congresso Brasileiro de Automática.

Toliyat, H. A. (1998). Analysis and simulation of five-phase variable-speed induction motor drives under asymmetrical connections, IEEE Trans. Power Electron. 13(4): 748-756.

Toliyat, H. A., Lipo, T. e White, J. C. (1991). Analysis of a concentrated winding induction machine for adjustable speed drive applications (parts i and ii), IEEE Trans. Energy Conversion 6(4): 679-692.
White, D. C. e Woodson, H. H. (1959). Electromechanical Energy Conversion, John Wiley and Sons, New York, NY. 